\title{
Vibration control systems for sensitive equipment: Limiting performance and optimal design
}

\author{
V.M. Ryaboy \\ Newport Corporation, 1791 Deere Avenue, Irvine, CA 92606, USA \\ Tel.: +1 949253 1866; Fax: +1949 253 1800; E-mail: vryaboy@newport.com
}

\begin{abstract}
As vibration control requirements become increasingly stringent, designers and users of vibration control equipment turn to devices and systems combining various physical mechanisms. Subsystems based on different physical effects can be combined to achieve the optimal performance for the application. Building an optimal product line that would cover a wide field of applications by combining several products, as opposed to creating one optimal device for a particular application, presents an optimum vibration control problem. This paper reviews optimum vibration control problems based on the idea of limiting performance, and discusses recent development of vibration control devices.
\end{abstract}

Keywords: Vibration control, vibration isolation, damping, shock isolation, passive isolation, active isolation, active damping, optimization, limiting performance

\section{Introduction}

Limiting performance analysis was first introduced by Sevin and Pilkey in [17-19] as a generalization of the traditional parametric optimization of shock and vibration isolation systems. A general dynamic system was considered as consisting of "structural elements" and "isolation elements". The isolator forces were considered to be explicit functions of time. These functions were then synthesized in order to minimize the performance index and satisfy the constraints. The process was called time-optimal synthesis. The resulting value of the performance index is the best that can be achieved by any isolator regardless of the hardware device utilized. The relationship between the optimal performance index and the constraints was termed the limiting performance characteristic [19]. Further developments along this line of research encompassed a wide class of problems as exemplified by the papers [9] and [21]. A detailed review can be found in the recent paper [2] and the monograph [3] by Balandin, Bolotnik and Pilkey.

These limiting performance problems seek to find the performance limitations stemming from the nature of the object being protected, while the means of control are not specified but rather described by a control function.

Generally speaking, the limiting performance investigation implies an estimate of the best results that can be obtained in a certain wide class of systems subject to a class of conditions. The result describes the limitations resulting from basic physical principles only. The technical means of building the protecting structures that would achieve this result are left to the ingenuity of the designer. 

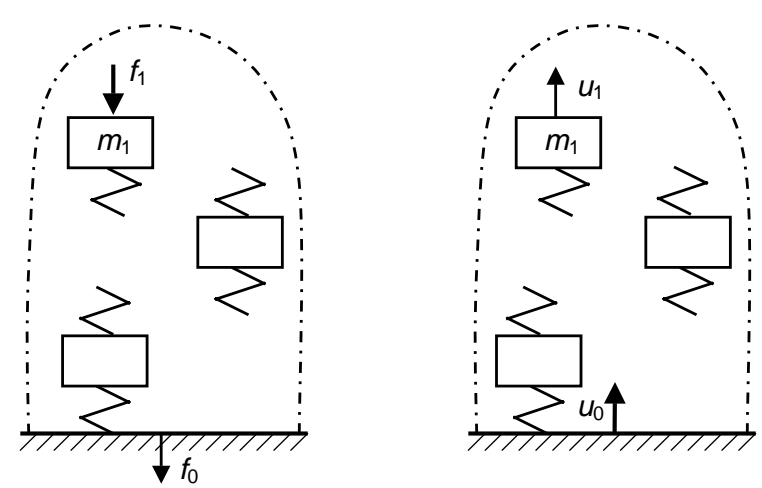

Fig. 1. Generic elastic-inertial passive isolation system. (a) Force excitation. (b) Kinematic excitation.

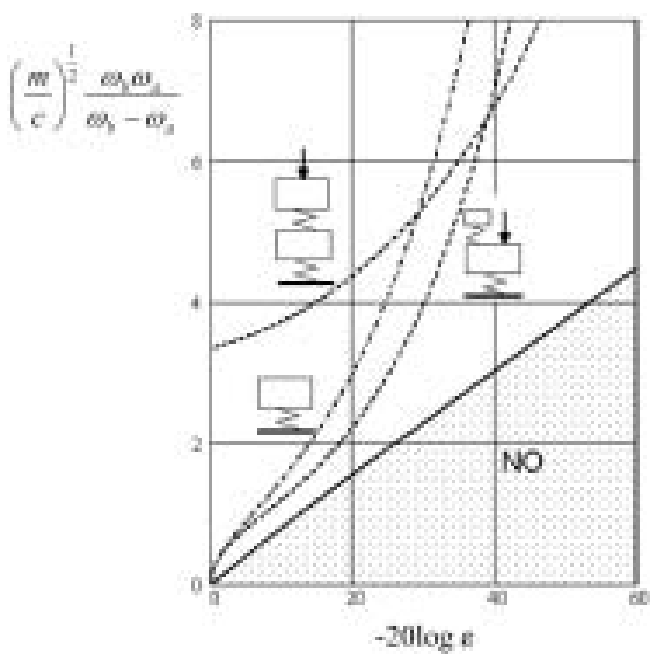

Fig. 2. Limiting performance of linear elastic-inertial vibration isolation systems.

\section{Limiting performance of passive elastic-inertial isolation systems}

A limiting performance formulation can be used to find the physical restrictions imposed by the requirement that the protecting structures belong to a certain category. This approach was originated at the end of the 1970's, when the efforts were aimed at distinguishing between the capabilities of the traditional passive vibration isolation systems and those of the new actively controlled systems. The goal was to prove that there is a physical limit to the performance of the whole class of multi-degree-of-freedom passive vibration isolation systems. Some of the results of this approach are reviewed here.

A generic passive vibration isolation system is defined as a system of material points elastically attached to each other and to a rigid foundation (see Fig. 1). The quality of isolation is characterized by the transmissibility $T(\omega)$, that is, the ratio of the force transmitted to the foundation, $f_{0}$, to the excitation force at the input point $m_{1}$, or, equivalently, the ratio of the amplitude at $m_{1}$ to the amplitude of kinematic excitation at the foundation. The vibration isolation requirement in a certain frequency domain, $\Omega$, can be stated as

$$
|T(\omega)| \leqslant \varepsilon(\omega), \quad \omega \in \Omega
$$

where $\varepsilon(\omega)$ is a prescribed function, $0<\varepsilon(\omega) \leqslant 1$. It was shown in [11] that for all linear conservative mechanical systems the following inequality relates the total mass of the system, $m$, and its overall static stiffness, $c$ (the ratio of static force at $m_{1}$ to the static displacement at this point), to the isolation performance: 


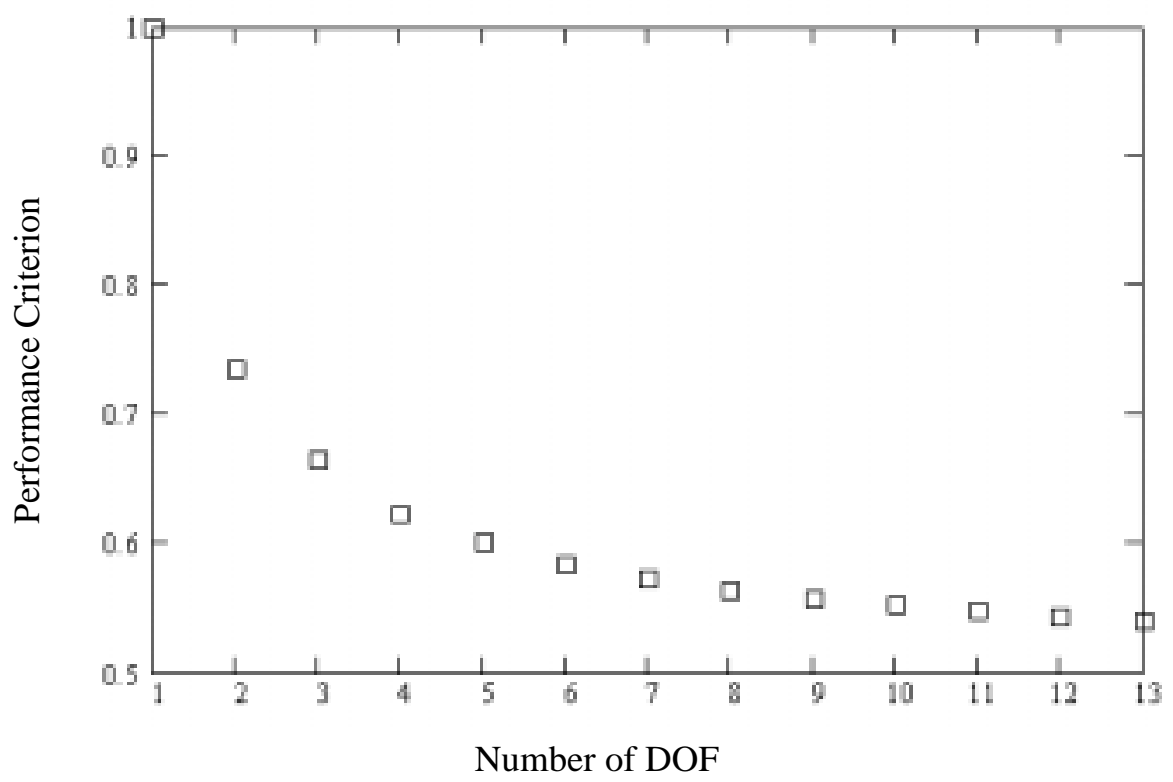

Fig. 3. Limiting performance of linear elastic-inertial shock isolation systems.

$$
\sqrt{\frac{m}{c}} \geqslant \frac{2}{\pi} \int_{\Omega}\left(\ln \frac{\pi}{\varepsilon(\omega)}-1\right) \frac{d \omega}{\omega^{2}}+\alpha
$$

where $\alpha$ is a known small residual term. Figure 2 represents this inequality graphically for constant $\varepsilon$, and $\Omega$ consisting of one interval, $\omega_{a} \leqslant \omega \leqslant \omega_{b}$. The area below the solid line on this graph corresponds to requirements that cannot be met by any linear conservative system. The dashed lines show the limiting capabilities of particular dynamic systems. Systems that approach the solid line from above can be synthesized as shown in $[8,13]$. Other limiting performance problems for the same class of systems were solved in $[12,13]$. Note that this limiting performance estimate confirms, in general, that the isolation task becomes more challenging not only for higher performance requirements, but also in the low-frequency domain.

The same approach was applied to shock isolation. As explained in [19], the best performance of an isolation system under an instantaneous shock represented by a velocity step $v_{0}$ is given by the relationship

$$
u_{0} w_{0}=\frac{1}{2} v_{0}^{2},
$$

where $u_{0}$ is the maximum relative displacement of the protected object, and $w_{0}$ is the maximum deceleration. The optimal control strategy in this case is to apply a constant resistance force (step response). The limiting quality of Eq. (1) can be achieved by a number of nonlinear and active single-degree-of-freedom systems. A class of non-linear control laws that achieve the optimal performance of Eq. (1) was described by [4] (see also Balandin et al. [3]). Bolotnik considered all isolators with power law characteristics in terms of displacement and velocity, and found only four types of isolators achieving the performance of Eq. (1). Only one out of the four was asymptotically stable: the isolator consisting of a linear spring and a quadratic damper.

In [15] it was shown that a linear multi-degree-of-freedom system can also approach the limiting quality of Eq. (1). Figure 3 plots the performance criterion, $u_{0} w_{0} / v_{0}^{2}$, vs. the number of degrees-of-freedom, $n$, for the optimal linear low-damped shock isolation systems of Fig. 1(b), where $m_{1}$ represents the object to be protected. The criterion decreases from 1 to the optimum value of $1 / 2$ as $n \rightarrow \infty$. Figure 4 shows how the reaction of the system approaches the optimal constant force response (step response) as $n$ increases. 

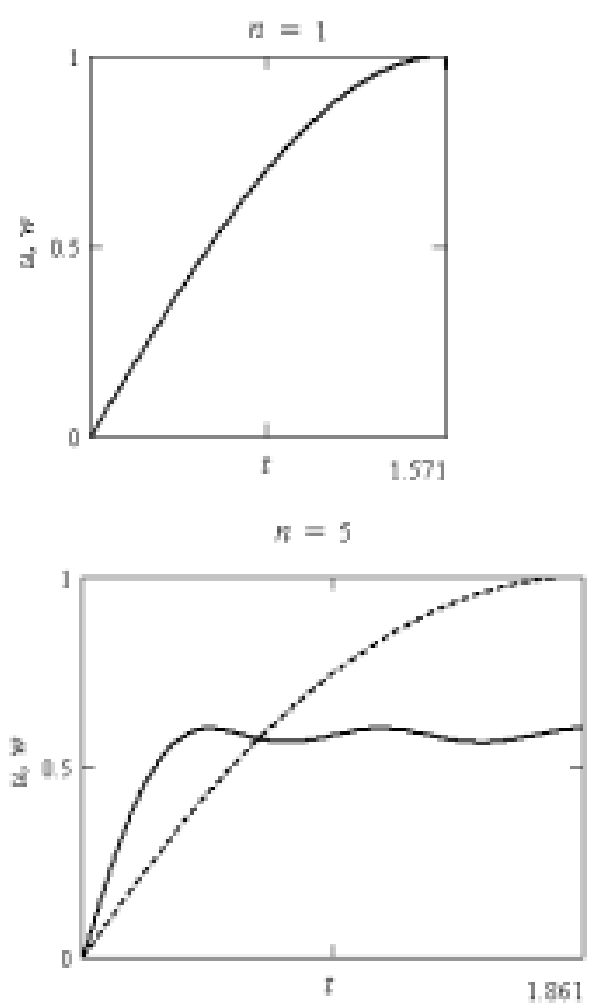
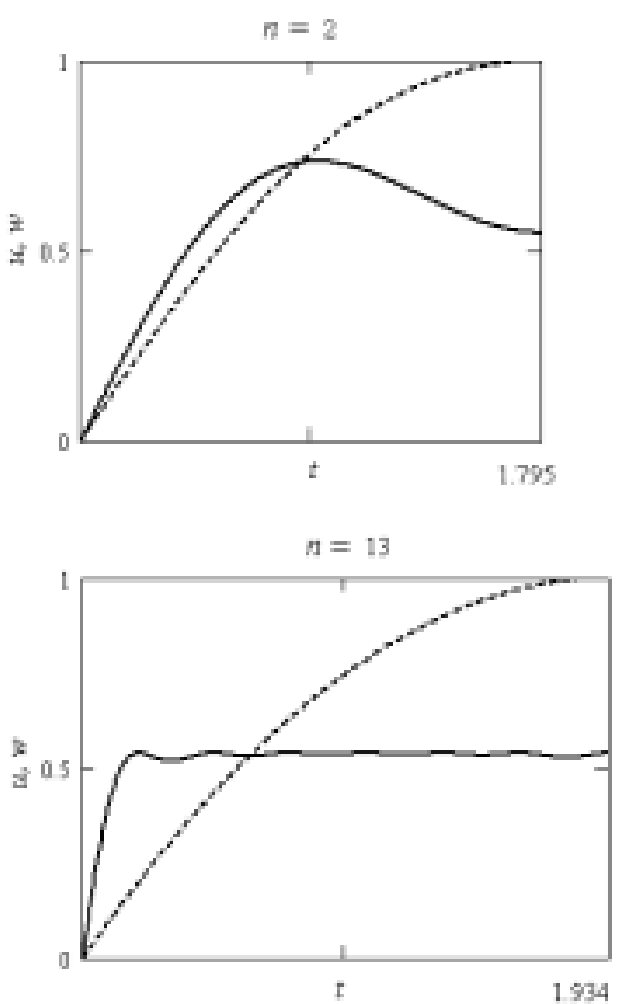

Fig. 4. Normalized relative displacement and deceleration time histories for the optimal linear elastic-inertial shock isolation systems. Dashed lines - displacement, solid lines - deceleration.

\section{Limiting performance of active elastic-inertial isolation systems}

Active vibration control systems are usually defined as systems including control forces driven by independent sources of energy. Figure 5 shows the schematic of a generic multi-degree-of-freedom active vibration control system attached to a rigid foundation. This model assumes that active forces are internal to the whole mechanical system including the foundation. Although the active systems can be much more efficient than the passive ones, they have their own physical limitations. Those limitations were found [14] in terms of the bounds of the work performed by active forces on system displacements. This work was adopted as a measure of the control effort. The normalized value of this work is given by $U_{0}=\left(\mathbf{f}^{\mathrm{a}}\right)^{\mathrm{T}} \mathbf{u} / f_{1}^{2}$ for the force excitation shown in Fig. 5(a), or by $U_{0}=\left(\mathbf{f}^{\mathrm{a}}\right)^{\mathrm{T}}\left(\mathbf{u}-u_{0} \mathbf{e}\right) / u_{0}^{2}$, where $\mathbf{e}=(1, \ldots, 1)^{\mathrm{T}}$, for the kinematic excitation shown in Fig. 5(b). The extrema of $U_{0}$ as a function of the active force vector, $\left(\mathbf{f}^{\mathrm{a}}\right)$, were determined at each frequency $\omega$, constrained by equations of motion and by the full compensation requirement ( $f_{0}=0$ for force excitation or $u_{1}=0$ for kinematic excitation). This resulted in explicit expressions for the extrema of $U_{0}$ in terms of the input-output transfer functions of the passive system only [13,14]. The qualitative graphs of those functions are presented in Fig. 6. Note that the work of active forces is always bounded from below by a positive number in some low frequency domain, and that no passive subsystem can produce a positive work on the system displacements.

\section{Limits of performance for linear controller design}

The actual design of the control loops, which had been set aside in the limiting performance analysis of active isolation systems presented above, is a challenging problem by itself. The linear controller design was addressed in terms of limiting performance by Boyd and Barratt [6] (see also Boyd et al. [5]). 


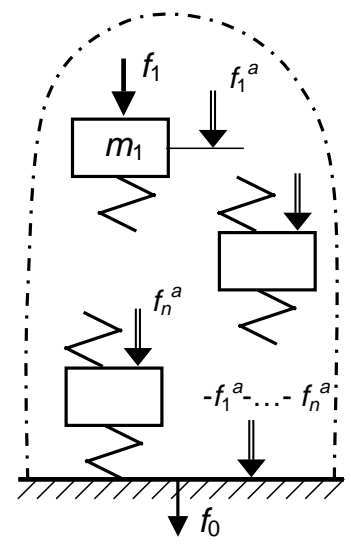

(a)

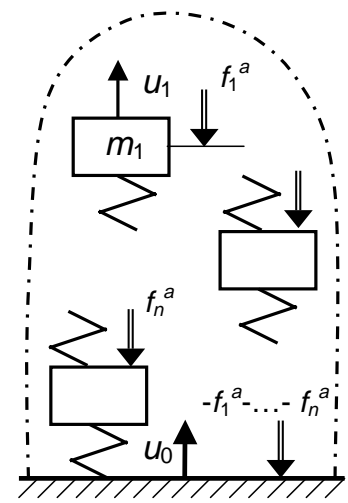

(b)

Fig. 5. Generic multi-degree-of-freedom elastic-inertial active vibration isolation system. (a) Force excitation. (b) Kinematic excitation.

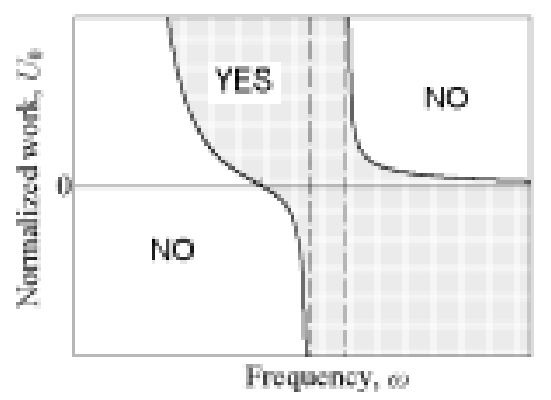

(a)

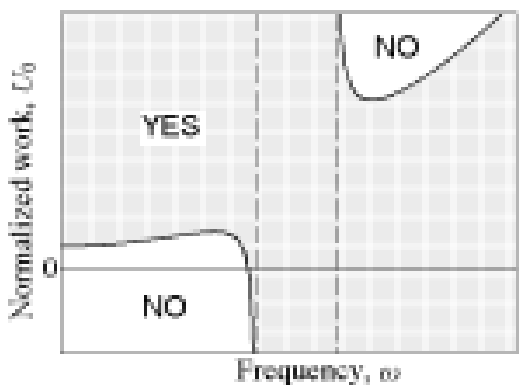

(b)

Fig. 6. Limiting performance of multi-degree-of-freedom active isolation systems. (a) Force excitation. (b) Kinematic excitation.

The following fundamental problem is addressed in [5,6]: given a specific system to be controlled, control configuration, and a set of design specifications, either find a control law that meets the given design specifications, or determine that none exists. Instead of conducting traditional parametric optimization, a limiting performance approach is taken, namely, the closed-loop system behavior is designed in terms of closed-loop transfer matrix $H(\omega)$, and only then is the control law determined that yields this system behavior

In this approach, the model of the plant includes more details about the system than is common in classical control. The model explicitly includes all the inputs and regulated variables that can influence a constraint or a specification. The authors show that the most common limitations and criteria applicable to the linear controller design, such as closed-loop stability, realizability, actuator authority and robustness, can be formulated in terms of 


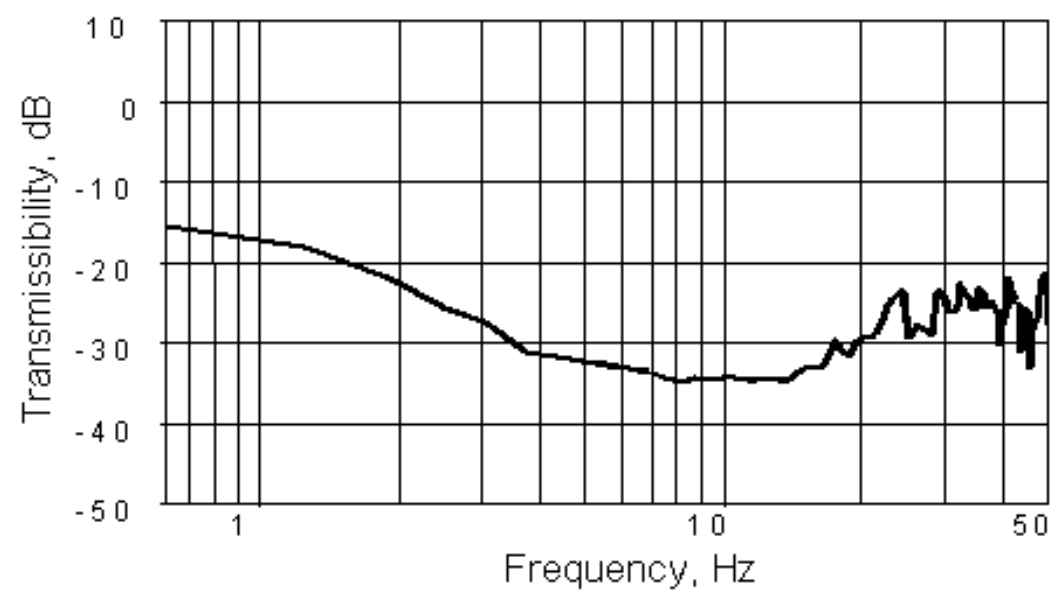

Fig. 7. Experimental transmissibility of the active vibration isolation system in the vertical direction.

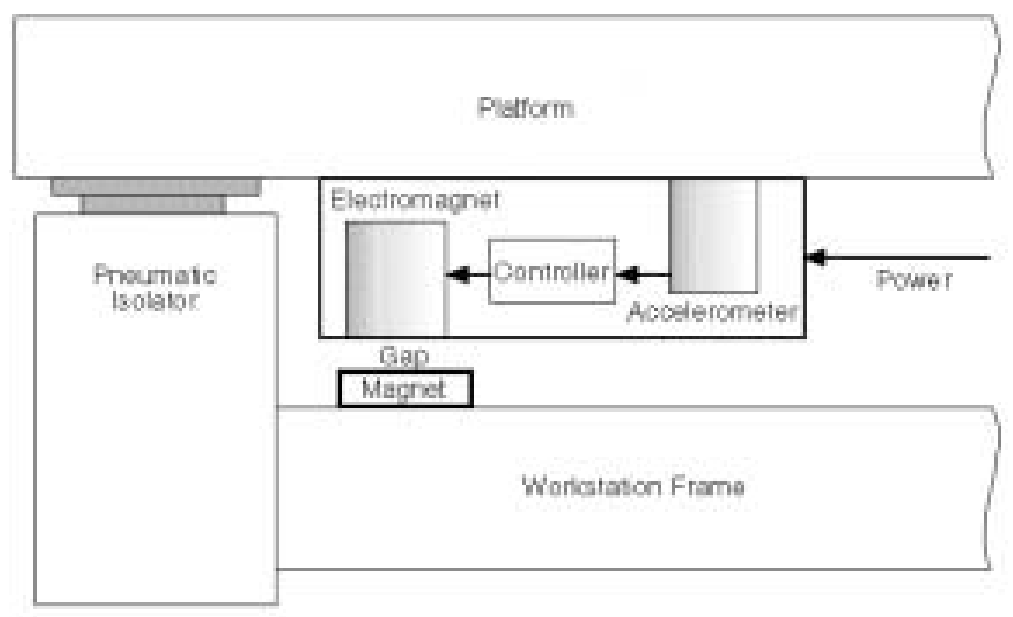

Fig. 8. Design and installation of the electromagnetic damper in a pneumatic workstation.

convex limitations on the transfer matrix $H(\omega)$. This allows for application of well-developed converging convex optimization algorithms. Numerous examples and a detailed bibliography on this subject can be found in $[5,6]$.

\section{Combination vibration control systems}

Shock and vibration isolation is a well-developed engineering discipline. The engineers practicing this discipline strive to make their designs optimal according to specific criteria, and optimization procedures pervade the design process of modern vibration control products. This discussion is limited to a particular field of shock and vibration control, specifically the platforms for vibration sensitive equipment commonly known as optical tables or workstations. However, those platforms can support any kind of vibration-sensitive payloads such as scientific research, optoelectronics or semiconductor equipment.

The engineers designing shock and vibration isolation products face numerous constraints and specifications that would ensure adequacy of their products in a wide range of applications. For example, in designing vibration isolation 


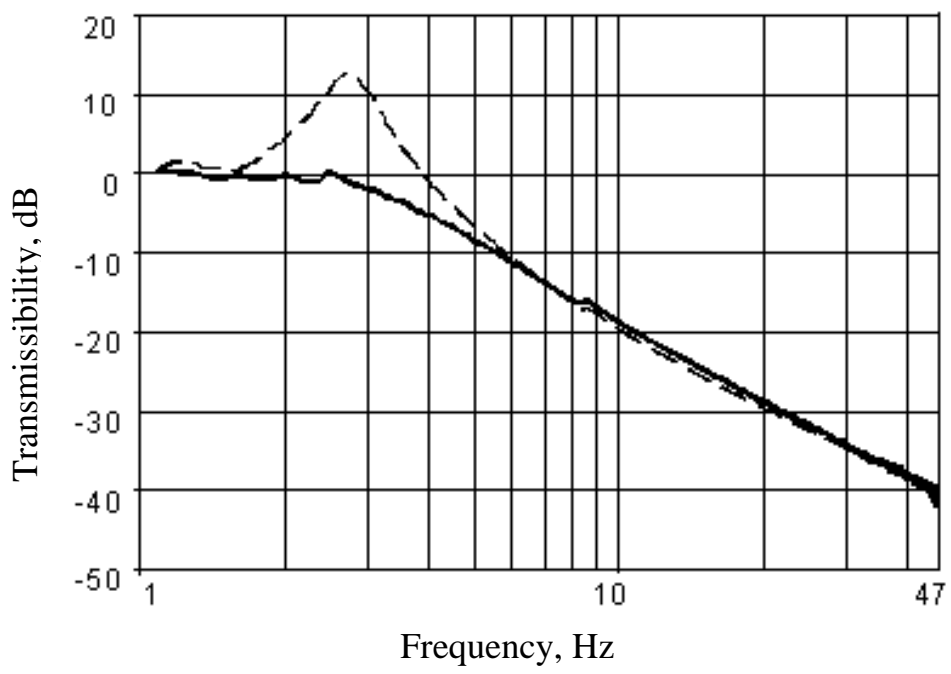

Fig. 9. Transmissibility of the pneumatic workstation with add-on active dampers. Dashed line - dampers off, solid line - dampers on.

systems for high precision vibration-sensitive equipment [10,20], the most important design requirements include load capacity, dynamic range of vibration input and vibration isolation performance. Detailed specifications include natural frequencies, allowable amplification at resonance, transient attenuation rate or "settling time", positional repeatability, recovery time after high-amplitude "saturating" event, control effort for active systems, etc.

Some of these criteria are interdependent. For example, the isolation efficiency of linear passive systems is determined mainly by the natural frequency, and the settling time is related to the resonance amplification since both are defined by damping. For non-linear or active systems the relationships between the criteria are more complicated.

In order to meet or optimize these criteria, various elements are employed in vibration isolation systems. Elastomeric elements are generally the most popular. Modern synthetic elastomers provide high damping, which ensures low resonance amplification and short settling time. A constant natural frequency (CNF) property, which is necessary in many applications, can be achieved by design [10]. The natural frequency, however, can hardly be lower than 5-8 Hz due to stability concerns and large displacements involved.

The pneumatic isolator is a "workhorse" of precision vibration control. The properties of pneumatic chambers can be found in [3] and [10]. Since the stiffness of a pneumatic chamber increases almost linearly with load, pneumatic isolators naturally possess the CNF property. Simple leveling valves allow them to maintain the same position independent of the load. These most sought after features allow pneumatic isolation systems to meet the stringent requirements of numerous modern applications. For isolation in horizontal directions, pneumatic isolators usually include pendulums or other mechanical decoupling devices [10]; oil is employed for damping horizontal vibrations. However, high-quality low-frequency pneumatic isolators sometimes do not meet stringent requirements of short settling time and low resonance amplification. A combination of a pneumatic chamber and an elastomeric element was suggested [16] in order to reduce the settling time of horizontal vibration. Another way to provide the necessary damping will be discussed in the next section.

Active isolation systems offer a significant increase in performance, especially at low frequencies. An active isolation system based on a combination of the piezoelectric controllable actuator and an elastomeric element for supporting the load and vertical isolation is described in [1]. The control loop transfer function is designed to compensate for the floor motion. To ensure a uniform efficiency in a wide frequency range, the control function and the elastomer parameters are optimized so that the controls compensate for the resonance caused by the elastomer. To make it possible in a robust controller configuration, a CNF elastomer is used. The result is a uniform high isolation starting from sub-Hertz domain as illustrated by experimental transmissibility in Fig. 7 . The isolation in the horizontal direction, provided by a traditional viscously damped pendulum, is lower than in the vertical direction, and the settling time after a horizontal perturbation may be not short enough. The subsequent development of this system is described in the next section in context of the optimum design of a product line. 

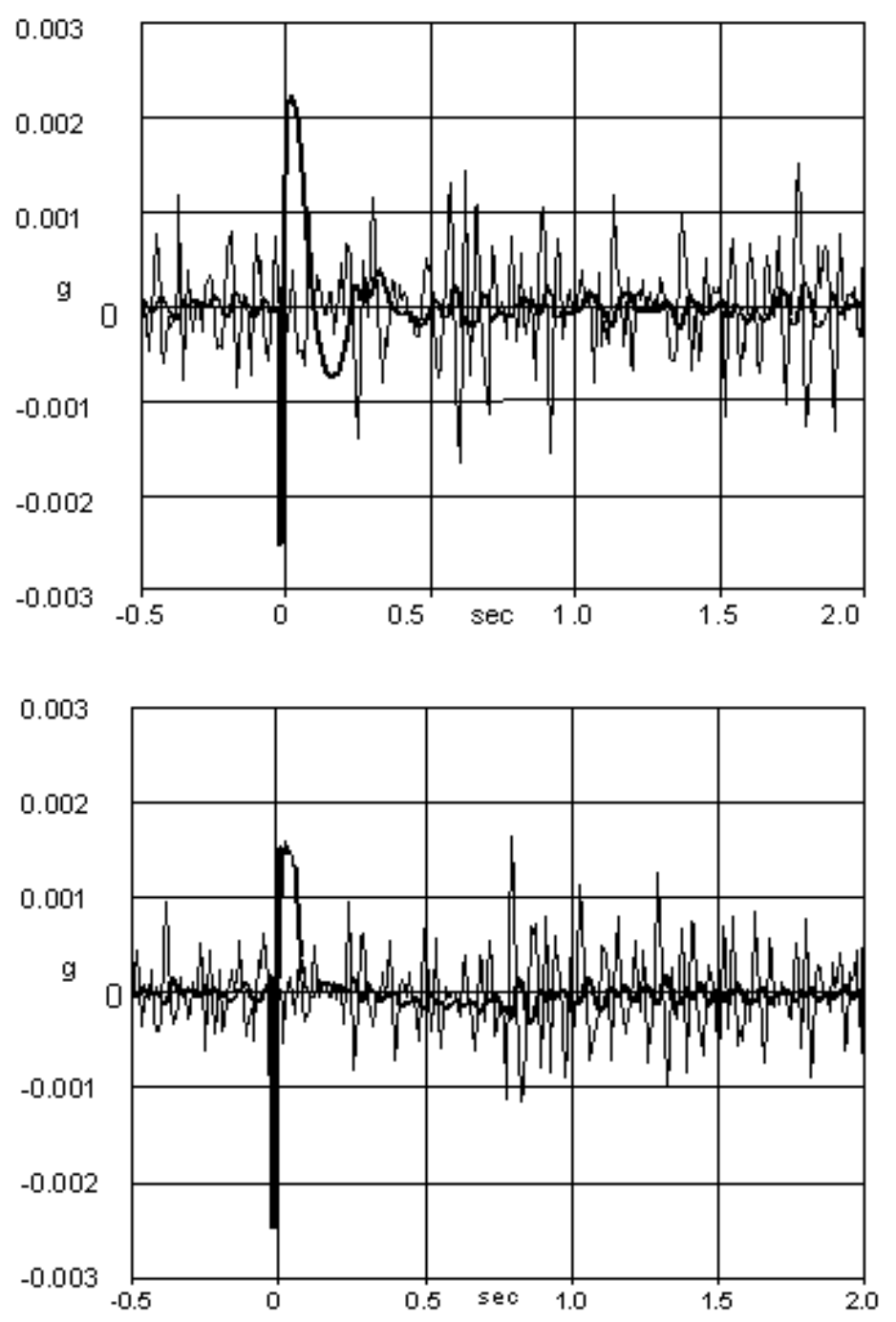

Fig. 10. Acceleration time history of a pneumatic workstation with add-on active dampers. Excitation by a short pulse. Top - dampers off; bottom - dampers on. Light traces show the floor vibration.

\section{Optimum design of a product line}

In the practical product development environment, the goal of the designer is to create a product or a set of products that would be adequate in numerous existing and future customer applications. The challenge is to build a portfolio of a limited number of products and technologies that would serve a maximum variety of customer applications. This can be formulated as an optimization procedure aimed at building a system of products and technologies that complement and combine with each other to cover a wide range of applications.

A viable formulation would be as follows: For the given $n$ existing products and $m$ performance criteria, define a new product which would be used in conjunction with the existing products and would cover the widest possible field of applications defined by these criteria. This formulation, of course, will not be rigorous. The detailed quantitative statement of the problem will not be attempted here since it would rely heavily on economic and marketing criteria that are outside the scope of this paper. Instead, the thesis is illustrated below by a case study showing how a new product augmented the performance of several existing products and thereby expanded their areas of applicability. 


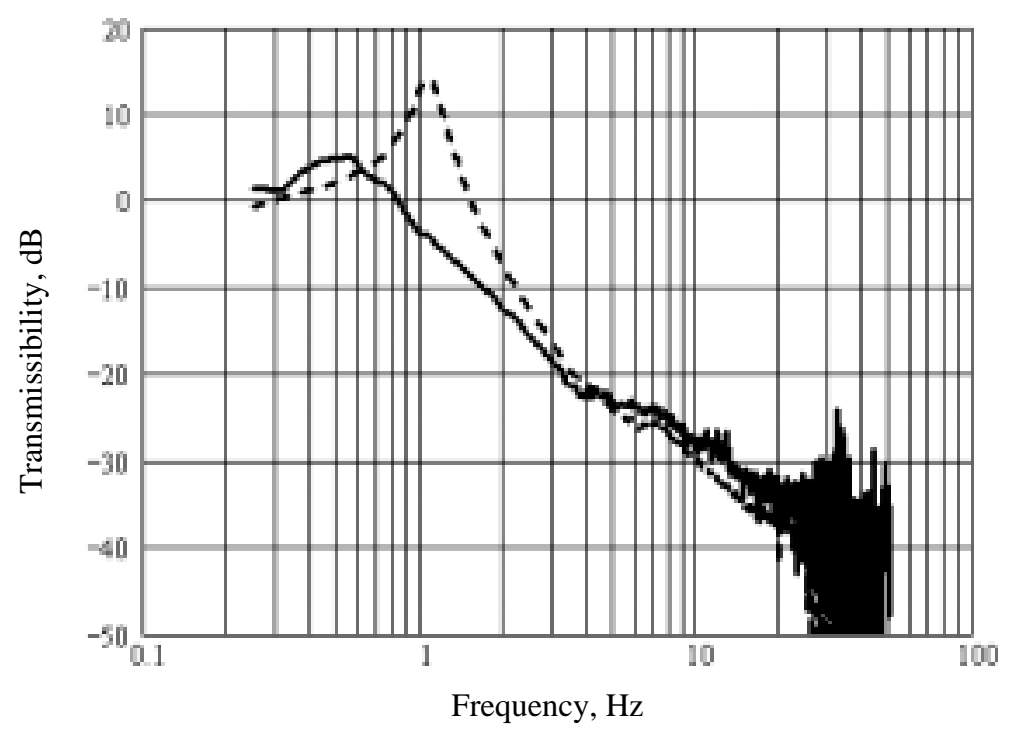

Fig. 11. Horizontal transmissibility of the actively isolated workstation with add-on active dampers. Dashed line - dampers off, solid line dampers on.

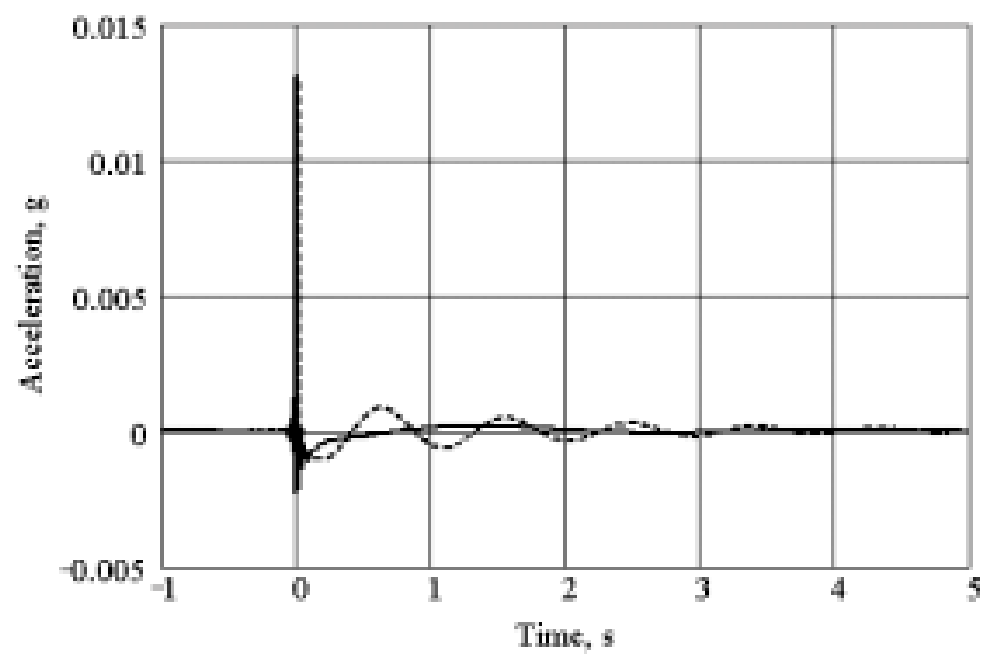

Fig. 12. Horizontal acceleration time history of the actively isolated workstation with add-on active dampers. Excitation by a short pulse. Dashed line - dampers off, solid line - dampers on.

An electromagnetic damper [7] was designed to be used as an add-on product with vibration isolators. Figure 8 shows the design schematics of the damper. Three dampers are normally used per workstation to damp vertical vibration. Figure 9 shows how the active vibration dampers eliminate the resonance vibrations of a pneumatic workstation. Figure 10 illustrates the improvement in settling time. The same damper model can be used with a variety of pneumatic isolators to increase damping of both vertical and horizontal vibrations without deterioration of isolation performance.

The same damper was also coupled with the active vibration isolator described in [1] to improve the horizontal transmissibility and the settling time. The experimental results are shown in Figs 11 and 12. The resulting system is actively controlled in all directions and provides high isolation performance due to a combination of piezoelectric active vibration compensation, passive isolation by elastomer and pendulum, viscous damping by oil, and 
electromagnetic active damping.

It can be concluded that the addition of an active electromagnetic damper to a line of vibration isolation products can enhance the performance of several isolators by eliminating low frequency resonances and reducing settling times after shock excitations. As a result, the area of application will expand to include payloads sensitive to low frequency vibration and payloads experiencing internal pulse excitation due to moving components such as stages.

\section{Conclusions}

Limiting performance solutions provide physical understanding and suggest practical solutions for shock and vibration isolation. New limiting performance problems for optimal shock and vibration isolation have been formulated and solved in recent years. The paper shows how the limiting performance approach applies to various aspects of the optimal shock and vibration control design process.

Combination of elements and products based on various physical phenomena is a way to address the wide variety of applications. It is also an approach to the optimal performance. A combination of vibration control devices can be used in designing an optimal system to cover a wide range of applications. This new optimal vibration control problem currently awaits theoretical formulation and solution.

\section{Acknowledgements}

This paper includes illustrations and experimental results by Newport Corporation. The author is grateful to his colleagues at Newport Corporation for their help and support.

\section{References}

[1] E.H. Anderson and B. Houghton, ELITE-3 active vibration isolation workstation, in: Smart Structures and Materials: Industrial and Commercial Applications of Smart Structures Technologies, (Vol. 4332), A.-M.R. Gowan, ed., Proceedings of the SPIE, 2001, pp. 183-196.

[2] D.V. Balandin, N.N. Bolotnik and W.D. Pilkey, Optimal protection from impact shock: theory and methods, Applied Mechanics Reviews 53(9) (2000), 237-264.

[3] D.V. Balandin, N.N. Bolotnik and W.D. Pilkey, Optimal Protection from Impact, Shock and Vibration, Gordon and Breach Science Publishers, Amsterdam, 2001.

[4] N.N. Bolotnik, Optimization of Amortization Systems (in Russian), Nauka, Moscow, 1983.

[5] S.P. Boyd, C. Barratt and S. Norman, Linear controller design: Limits of performance via convex optimization, Proceedings of the IEEE 78 (1990), 529-574.

[6] S.P. Boyd and C. Barratt, Linear Controller Design: Limits of Performance, Prentice Hall, Englewood Cliffs, NJ, 1991.

[7] L.P. Fowler, S. Buchner and V.M. Ryaboy, Self-contained active damping systems for pneumatic isolation tables, in: Smart Structures and Materials: Industrial and Commercial Applications of Smart Structures Technologies, (Vol. 3991), J.H. Jacobs, ed., Proceedings of the SPIE, 2000, pp. 261-272.

[8] M.D. Genkin and V.M. Ryaboy, Elastic-Inertial Vibration Isolation Systems: Limiting Performance, Optimal Configurations (in Russian), Nauka, Moscow, 1988.

[9] W.D. Pilkey and L. Kitis, Limiting performance of shock isolation systems by modal approach, Earthquake Engineering and Structural Dynamics 14 (1986), 75-81.

[10] E.I. Rivin, Passive Vibration Isolation, ASME Press, New York, 2003

[11] V.M. Ryaboy, Limiting capabilities of elastic-inertial vibration-proofing, Mechanics of Solids 17(5) (1982), 37-44.

[12] V.M. Ryaboy, Limit of effectiveness of elastic-inertial vibration-isolating systems below and above resonance, Mechanics of Solids 28(1) (1993), 39-46.

[13] V.M. Ryaboy, Limiting performance and optimal synthesis of the vibration isolating structures, in: Structural Dynamics: Recent Advances, Proceedings of the 5th International Conference, (Vol. 2), N.S. Ferguson, ed., Institute of Sound and Vibration Research, Southampton, UK, 1994, pp. 681-698.

[14] V.M. Ryaboy, Limiting performance estimates for the active vibration isolation in multi-degree-of-freedom mechanical systems, Journal of Sound and Vibration 186 (1995), 1-21.

[15] V.M. Ryaboy, Shock isolation by a low-damped multi-degree-of-freedom mechanical system, Journal of Sound and Vibration 197 (1996), 381-385.

[16] V.M. Ryaboy, W.B. Houghton and H.B. Keil, Pneumatic vibration isolator utilizing an elastomeric element for isolation and attenuation of horizontal vibration, US Patent 6, 619, 611, 16 September, 2003. 
[17] E. Sevin, Min-max solutions for the linear mass-spring system, Journal of Applied Mechanics 24 (1957), 131-136.

[18] E. Sevin and W.D. Pilkey, Computational approaches to minmax response of dynamic systems with incompletely prescribed input functions, Journal of Applied Mechanics 34 (1967), 87-90.

[19] E. Sevin and W.D. Pilkey, Optimum Shock and Vibration Isolation, SAVIAC, Washington, 1971.

[20] E.E Ungar, D.H. Sturz and C.H. Amick, Vibration control design of high technology facilities, Sound and Vibration 24(7) (1990), 20-25.

[21] B.P. Wang and W.D. Pilkey, Limiting performance characteristics of steady-state systems, Journal of Applied Mechanics 42 (1975), 721-726. 

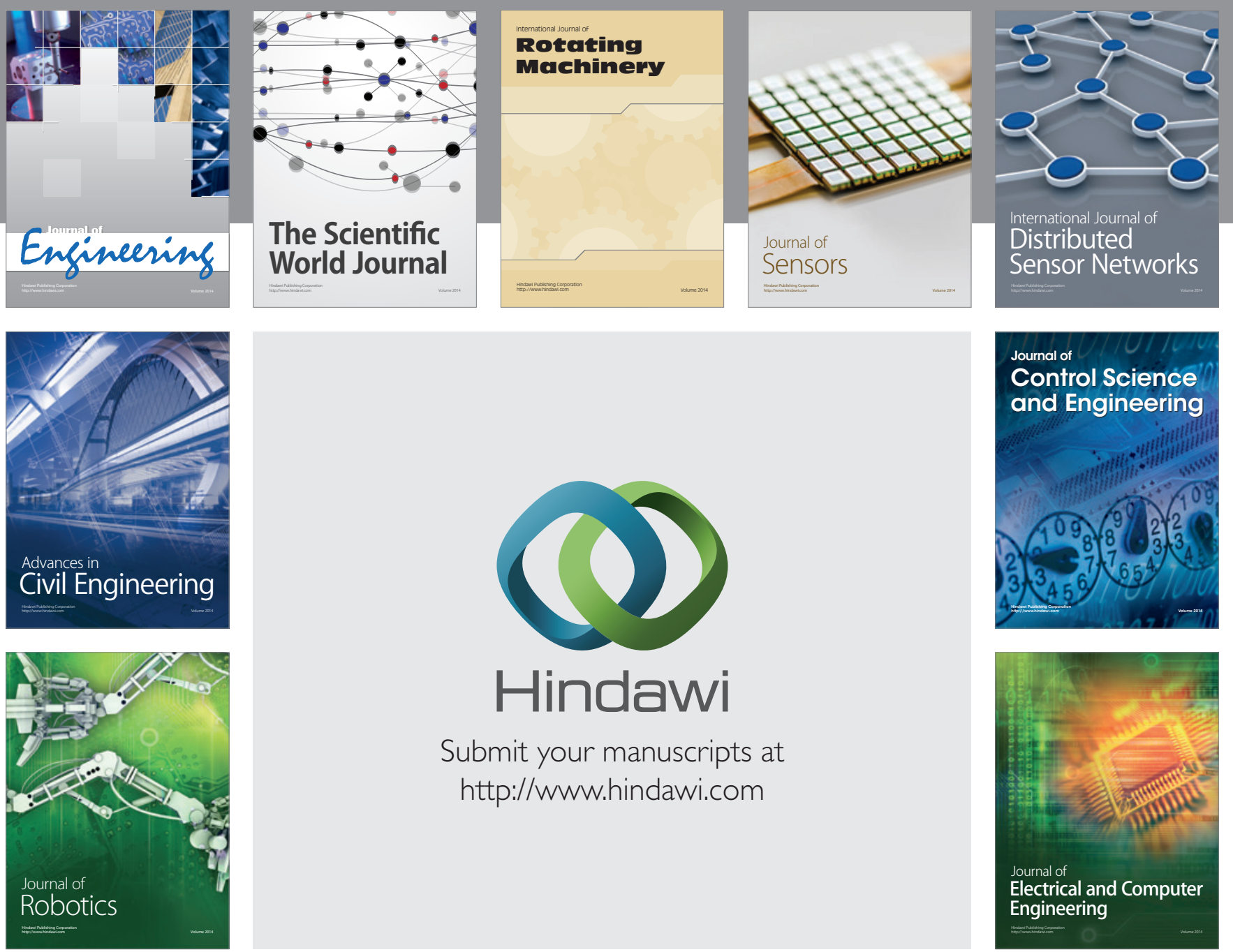

Submit your manuscripts at

http://www.hindawi.com
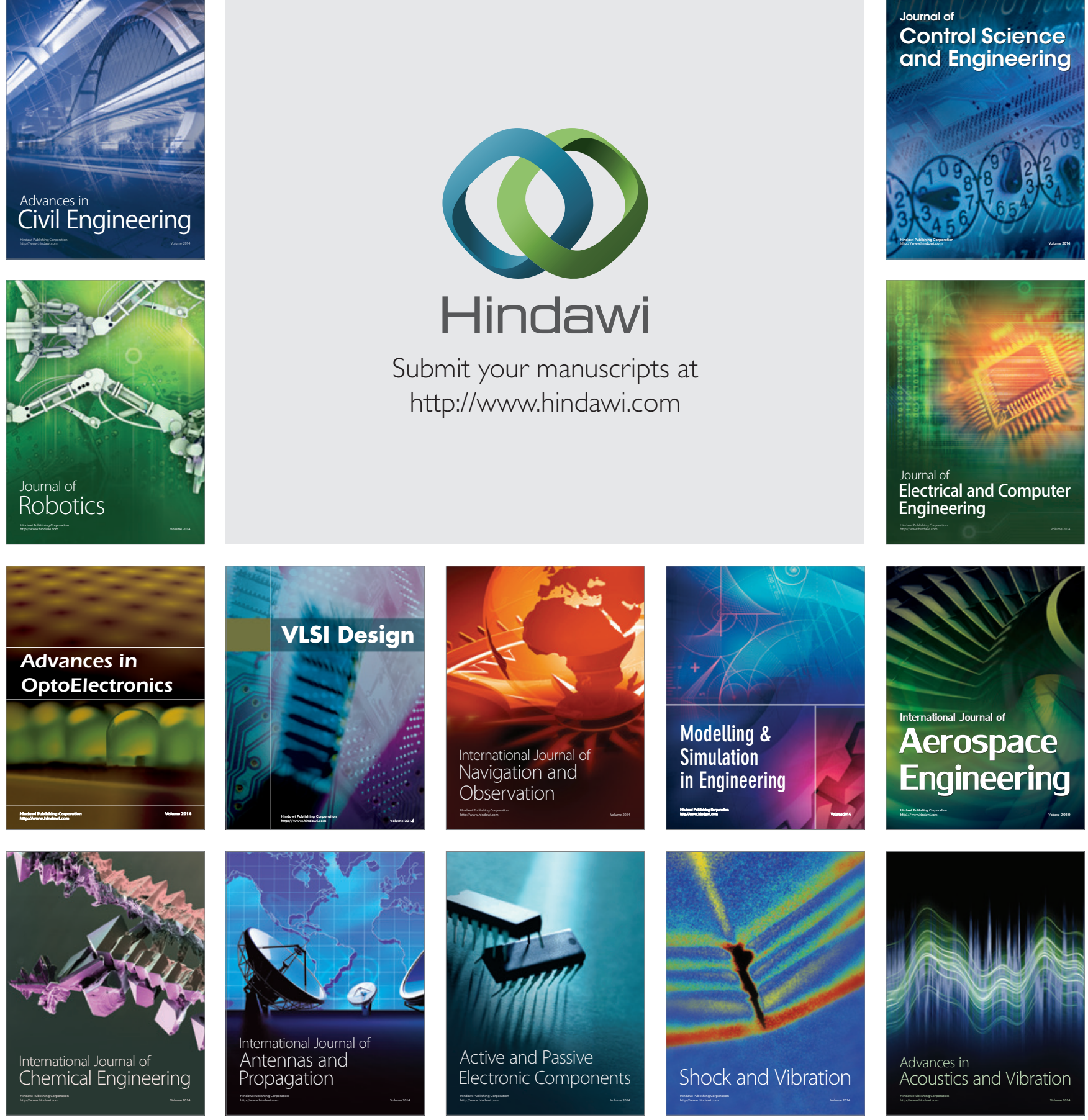